\title{
Questes
}

\section{Faire communauté : éléments bibliographiques}

Julia Conesa Soriano et Julie Pilorget

\section{(2) OpenEdition}

Journals

Édition électronique

URL : http://journals.openedition.org/questes/4328

DOI : 10.4000/questes.4328

ISSN : 2109-9472

\section{Éditeur}

Les Amis de Questes

\section{Édition imprimée}

Date de publication : 6 mai 2016

Pagination : 161-166

ISSN : 2102-7188

\section{Référence électronique}

Julia Conesa Soriano et Julie Pilorget, «Faire communauté : éléments bibliographiques », Questes [En ligne], 32 | 2016, mis en ligne le 10 mai 2016, consulté le 15 septembre 2020. URL : http:// journals.openedition.org/questes/4328

Ce document a été généré automatiquement le 15 septembre 2020.

(C) Association des amis de «Questes » 


\title{
Faire communauté : éléments bibliographiques
}

\author{
Julia Conesa Soriano et Julie Pilorget
}

\section{GÉNÉRALITÉS}

1 "Communes and community ", dans The Oxford Dictionary of Middle Ages, dir. Robert E. BJoRK, Oxford, Oxford University Press, 2010, 4 vol., t. II, p. 428.

2 MICHAUD-QUANTIN, Pierre, Universitas : expressions du mouvement communautaire dans le Moyen Âge latin, Paris, Vrin, 1970.

3 Vivre en société au Moyen Âge. Occident chrétien $\mathrm{VI}^{e}-\mathrm{XV} \mathrm{V}^{e}$ siècle, dir. Claude CAROZZI, Daniel LE BLEVEC et Huguette taVIANI-CAROzZI, Aix-en-Provence, Publications de l'Université de Provence, 2008.

\section{INDIVIDU, COMMUNAUTÉ ET EXCLUSION}

4 Exclure de la communauté chrétienne. Sens et pratiques sociales de l'anathème et de l' excommunication (IV $\left.V^{e}-X I I^{e} s.\right)$, dir. Geneviève BÜHRER-THIERRY et Stéphane GIOANNI, Turnhout, Brepols, 2015.

5 Exclus et systèmes d'exclusion dans la littérature et la civilisation médiévales. Sénéfiance, vol. 5, 1978.

6 L'Individu au Moyen Âge. Individuation et individualisation avant la modernité, dir. Brigitte Miriam BEDOS-REZAK et Dominique IOGNA-PRAT, Paris, Aubier, 2005.

7 IOGNA-PRAT, Dominique, Ordonner et exclure. Cluny et la société chrétienne face à l'hérésie, au judaïsme et à l'islam, 1000-1150, Paris, Aubier, 1998.

8 Minorités et régulations sociales en Méditerranée médiévale. Actes du colloque réuni du 7 au 9 juin 2007 en l'abbaye royale de Fontevraud, dir. Stéphane BOISSELIER, François CLEMONT et John TOLAN, Rennes, PUR, 2010. 

Imago, 1988. medioevo, 1991.

RoSENWEIN, Barbara H., "Y avait-il un “moi” au haut Moyen Âge ? », Revue historique, $\mathrm{n}^{\circ}$ 633, 2005, p. 31-52.

WALKER BYNUM, Caroline, "Did the twelfth century discover the individual ? ", Journal of Ecclesiastical History, vol. 31, janvier 1980, p. 1-17.

ZAREMSKA, Hanna, Les Bannis au Moyen Âge, Paris, Aubier, 1996.

\section{LES COMMUNAUTÉS RELIgIEUSES ET HOSPITALIÈRES}

BERIAC, Françoise, Histoire des lépreux au Moyen Âge, une société d'exclus, Paris, Éditions

BERIOU, Nicole et TOUATI, François-Olivier, Voluntate Dei leprosus. Les lépreux entre conversion et exclusion aux $\mathrm{XII}^{e}$ et $\mathrm{XIII}^{e}$ siècles, Spoleto, Centro italiano di studi sull'alto

CAPELLE, Catherine, Le Vœu d'obéissance des origines au XII siècle: étude juridique, Paris, R. Pichon et R. Durand-Auzias, 1959.

L'Encadrement religieux des fidèles au Moyen Âge et jusqu'au concile de Trente, Actes du $109^{e}$ Congrès des Sociétés savantes (Dijon,1984), dir. Congrès national des sociétés savantes (Section d'histoire médiévale et de philologie), Paris, Comité des travaux historiques et scientifiques, 1985.

PARISSE, Michel, Les Nonnes au Moyen Âge, Le Puy, Christine Bonneton éditeur, 1983.

SIMONS, Walter, Cities of ladies. Beguine communities in the medieval Low Countries. 1200-1565, Philadelphia, University of Pennsylvania Press, 2001.

Sous la règle de saint Benoît: structures monastiques et sociétés en France du Moyen Âge à l' époque moderne. Actes du colloque tenu à l'abbaye bénédictine Sainte-Marie de Paris (2325 octobre 1980), dir. Centre de recherches d'histoire et de philologie de la IV ${ }^{\mathrm{e}}$ section de l'École Pratique des Hautes Études, Genève/Paris, Droz/Champion, 1982.

19 La Vita comune del clero nei secoli XI e XII. Atti della settimana di studio: Mendola, settembre 1959, dir. Centro di studi medioevali, Milano, Vita e Pensiero, 1962.

\section{Les communautés religieuses laïques}

Les Confréries, l'Église et la Cité: cartographie des confréries du Sud-Est, dir. Marie-Hélène FROESCHLÉ-CHOPARD, Grenoble, Centre alpin et rhodanien d'ethnologie, 1988.

HANAWALT, Barbara, « Keepers of the lights : late medieval parish guilds », The Journal of Medieval and Renaissance Studies, vol. 14, 1984, p. 21-37.

2 LE BRAS, Gabriel, "Les confréries chrétiennes : problèmes et propositions ", Revue d' histoire du droit français et étranger, Série 4, vol. 19-20, 1940-1941, p. 310-363, repris dans Études de sociologie religieuse, Paris, PUF, 1955, p. 423-463.

MEERSSEMAN, Gilles Gérard, Ordo fraternitatis : confraternite e pietà dei laici nel medioevo, Roma, Herder, 1977, 3 vol.

RUBIN, Miri, Charity and community in medieval Cambridge, Cambridge, Cambridge University Press, 1987. 


\section{Les communautés confessionnelles et ethniques}

\section{Communautés juives} BARON, Salo Wittmayer, The Jewish Community. Its history and structure to the American revolution, Westport, Greenwood Press Publishers, 1972, 3 vol.

COULET, Noël, «De l'intégration à l'exclusion : la place des Juifs dans les cérémonies d'entrée solennelle au Moyen Âge ", Annales. Économies. Sociétés. Civilisations, vol. 34, n 4, juillet-août 1979, p. 676-683.

FINKELSTEIN, Louis, Jewish Self-Government in the Middle Ages, $2^{\mathrm{e}}$ édition, New York, Philipp Feldheim Inc., 1964.

GRABOÏs, Aryeh, «Remarques sur l'influence mutuelle de l'organisation de la communauté juive et de la paroisse urbaine dans les villes entre le Rhin et la Loire à la veille des croisades ", dans Le istituzioni ecclesiastiche della « societas christiana » dei secoli XI-XII : diocesi, pievi e parrocchie. Atti della sesta Settimana internazionale di studio (Milano, 1-7 sett. 1974), dir. Centro di studi medioevali, Milano, Vita e pensiero, 1977, p. 546-558.

PARKES, James, The Jew in the medieval community. A Study of his political and economic situation [1938], London, The Soncino Press , $2^{\mathrm{e}}$ édition avec une nouvelle introduction, New York, Hermon Press, 1976.

schWARzfuchs, Simon, Kahal, la communauté juive de l'Europe médiévale, Paris, Maisonneuve et Larose, 1986.

SHATZMILLER, Joseph, "L'excommunication, la communauté juive et les autorités temporelles au Moyen Âge ", dans Les Juifs dans l'histoire de France, dir. Myriam YARDENI, Leiden, E. J. Brill, 1980, p. 63-69.

SHATZMiLLER, Joseph, «L'organisation communautaire et les limites du "Self-Government " en Provence (1250-1350)», Cahiers de la Méditerranée: les Juifs dans la Méditerranée médiévale et moderne, Actes des journées d'études du C.M.M.C., 25-26 mai 1983, vol. 9, n ${ }^{\circ} 1$, 1986, p. 1-9.

\section{Autres communautés confessionnelles ou ethniques}

AILlET, Cyrille, Les Mozarabes. Christianisme, islamisation et arabisation en péninsule Ibérique, $\mathrm{IX}^{\mathrm{e}}-\mathrm{XII}^{\mathrm{e}}$ siècle, Madrid, Casa de Velázquez, 2010.

EDDÉ, Anne-Marie, MICHEAU, Françoise et PICARD, Christophe, Communautés chrétiennes en Pays d'Islam : du début du vII siècle au milieu du XI ${ }^{e}$ siècle, Paris, Sedes, 1997.

5 MAZZOLI-GUINTARD, Christine, "Mosquées, territoire et communauté du quartier en alAndalus : l'exemple de Cordoue aux $\mathrm{x}^{\mathrm{e}}-\mathrm{XI}^{\mathrm{e}}$ siècles ", dans Iglesias $y$ Fronteras. Homenaje a J. Rodriguez Molina, dir. Francisco TORO CEBAllos, Jaèn, Disputacion Provincial de Jaèn, 2005 , p. $465-480$. 


\section{LES COMMUNAUTÉS D'HABITANTS}

\section{Les communautés urbaines}

ARTIFONI, Enrico, "Città e comuni ", dans Storia medievale, dir. Enrico ARTIFONI et al., Roma, Donzelli, 1998, p. 363-386.

BOUCHERON, Patrick et MENJOT, Denis, «La ville médiévale », dans Histoire de l'Europe urbaine, dir. Jean-Luc PinoL, Paris, Le Seuil, 2003, t. 1, p. 287-592.

CHEVALIER, Bernard, Les Bonnes Villes de France du XIVe au XVI siècle, Paris, Aubier, 1982.

La Città nell'Alto Medioevo. Settimane di studio del Centro italiano di studi sull'alto Medioevo, VI, dir. Centro italiano di studi sull'alto medioevo, Spoleto, Centro italiano di studi sull' alto medioevo, 1959.

COULET, Noël, «Quartiers et communauté urbaine en Provence (XIII-XV siècles) », dans Villes, bonnes villes, cités et capitales. Études d'histoire urbaine $\left(\mathrm{XII}^{\mathrm{e}}-\mathrm{XVIII}{ }^{\mathrm{e}} \mathrm{s}\right.$.) offertes à Bernard Chevalier, dir. Monique BouRin, Caen, Paradigme, 1993, p. 351-359.

DUTOUR, Thierry, La Ville médiévale. Origines et triomphe de l'Europe urbaine, Paris, Odile Jacob, 2003.

HEERS, Jacques, La Ville au Moyen Âge en Occident: paysages, pouvoirs et conflits, Paris, Hachette, 1990.

KUCHENBUCH, Ludolf, MORSEL, Joseph et SCHELER, Dieter, « La construction processionnelle de l'espace communautaire ", dans Écritures de l'espace social médiéval. Mélanges offerts à Monique Bourin, dir. Didier Borsseuil et al, Paris, Publications de la Sorbonne, 2010, p. 139-182.

4 MORSEL, Joseph, «Appropriation communautaire du territoire, ou appropriation territoriale de la communauté ? Observations en guise de conclusion ", Hypothèses, $n^{\circ} 9$, 2006, p. 89-104.

5 MORSEL, Joseph, « Comment peut-on être Parisien ? Contribution à l'histoire de la genèse de la communauté parisienne au XIII ${ }^{\mathrm{e}}$ siècle ", dans Religion et société urbaine au Moyen Âge. Mélanges offerts à Jean-Louis Biget, dir. Patrick BOUCHERON et Jacques CHIFFOLEAU, Paris, Publications de la Sorbonne, 2000, p. 363-381.

Les Origines des libertés urbaines: actes du XVI Congrès des historiens médiévistes de l' enseignement supérieur, Rouen, 7-8juin 1985, dir. Société des historiens médiévistes de l'enseignement supérieur public, Mont-Saint-Aignan, Publications de l'Université de Rouen, 1990.

RICHÉ, Pierre, "La représentation de la ville dans les textes littéraires du $\mathrm{V}^{\mathrm{e}}$ au $\mathrm{IX}^{\mathrm{e}}$ siècles ", dans La Fin de la cité antique et le début de la cité médiévale. De la fin du III siècle à $l$ 'avènement de Charlemagne, dir Claude LEPELLEY, Bari, Edipuglia, 1996, p. 183-190.

La Ville au Moyen Âge. Actes du $120^{e}$ congrès national des sociétés historiques et scientifiques, Aix-en-Provence, 23-29 octobre 1995, dir. Noël coulet et Olivier GUYOTJEAnNIN, Paris, Éd. du C.T.H.S., 1999.

4 Villes, bonnes villes, cités et capitales. Études d'histoire urbaine offertes à Bernard Chevalier, dir. Monique BouRIN, Tours, Université de Tours, 1989.

Villes et sociétés urbaines au Moyen Âge: hommage à M. le professeur Jacques Heers, dir. Marie-Thérèse CARON et al., Paris, PUPS, 1994. 

siècle). Colloque organisé à l'Université libre de Bruxelles en décembre 2004, dir. Claire BILEN et Chloé DELIGNE, Turnhout, Brepols, 2007.

\section{Les communautés rurales}

ASSIER-ANDRIEU, Louis, « La communauté villageoise. Objet historique. Enjeu théorique », Ethnologie française, vol. 16, $\mathrm{n}^{\circ} 4,1986$, p. 351-360.

BLOCH, Marc, «Une nouvelle théorie sur l'origine des communes rurales », Annales d' histoire économique et sociale, vol. 1, 1929, p. 587-589.

BOURIN-DERRUAU, Monique, Villages médiévaux en Bas-Languedoc : genèse d'une sociabilité ( $X^{e}$ XIV siècles), Paris, L'Harmattan, 1987, 2 vol.

Des Paroisses de Touraine aux communes d'Indre-et-Loire. La formation des territoires, dir. Élisabeth ZADORA-RIO, Tours, FERACF, 2008.

Les Communautés rurales. 4, Europe occidentale: Italie, Espagne, France, dir. Société Jean Bodin pour l'histoire comparative des institutions, Paris, Dessain et Tolra, 1984.

Les Communautés villageoises en Europe occidentale du Moyen Âge aux Temps modernes. Quatrième journées internationales d'Histoire de l'abbaye de Flaran (8-10 septembre 1982), dir. Centre culturel de l'abbaye de Flaran, Auch, diffusion Comité départemental du tourisme du Gers, 1984.

FOSSIER, Robert, "Les “communautés rurales” au Moyen Âge », Journal des Savants, juillet-décembre 1992, p. 237-276.

LETURCQ, Samuel, «Espace du village, terrouers des hameaux. Théories et pratiques spatiales d'une communauté paysanne en Beauce orléanaise aux $\mathrm{XIV}^{\mathrm{e}}$ et $\mathrm{XV}^{\mathrm{e}}$ siècles ", dans Construction de l'espace au Moyen Âge : pratiques et représentations. XXXVII congrès de la S.H.M.E.S. (Mulhouse, 2-4 juin 2006), dir. Thomas LIENHARD, Paris, Publications de la Sorbonne, 2007, p. 229-241.

MORSEL, Joseph, " “Communautés d'installés”. Pour une histoire de l'appartenance médiévale au village ou à la Ville », EspacesTemps.net, 11.11.2014, http:// www.espacestemps.net/articles/communautes-dinstalles/

Mouthon, Fabrice, Les Communautés rurales en Europe au Moyen Âge: une autre histoire politique du Moyen Âge, Rennes, PUR, 2014.

Paysans en leurs communautés, Cahiers de recherche médiévale (XIII $-X V^{e}$ s.), vol. X, 2003.

SIVÉRY, Gérard, Terroirs et communautés rurales dans l'Europe occidentale au Moyen Âge, Lille, Presses universitaires de Lille, 1990.

Vivre au village au Moyen Âge. Les solidarités paysannes $d u \mathrm{XI}^{e}$ au XIII ${ }^{e}$ siècle, dir. Monique BOURIN et Robert DURAND, Rennes, PUR, 2000.

WICкнAм, Chris, Communautés et clientèles en Toscane au XII siècle. Les origines de la commune rurale dans la région de Lucques, (trad. française de Communità e clientele, 1995), Rennes, Association d'Histoire des Sociétés Rurales, 2001. 


\section{LES COMMUNAUTÉS DE MÉTIERS}

BLACK, Anthony, Guilds and civil society in European political thought from the twelfth century to the present, London, Methuen, 1984.

CHEVALIER, Bernard, «Corporations, conflits politiques et paix sociale en France aux XIV et $\mathrm{XV}^{\mathrm{e}}$ siècles ", Revue historique, vol. 543, juillet-septembre 1982, p. 17-44.

COORNAERT, Émile, Les Corporations en France avant 1789, Paris, Gallimard, 1941.

DEGRASSI, Donata, L'Economia artigiana nell'Italia medievale, Roma, La Nuova Italia Scientifica, 1996.

EPSTEIN, Steven A., Wage, labor and guilds in medieval Europe, Chapel Hill, University of North Carolina press, 1991.

EPSTEIN, Steven A., "Craft guilds, apprenticeship and technological change in preindustrial Europa », Journal of economic history, vol. 58, $\mathrm{n}^{\circ} 3,1998$, p. 684-713.

Gilde und Korporation: in den Nordeuropäischen Städten des späten Mittelalters, dir. Klaus FRIEDLAND, Köln, Böhlau, 1984.

GOURON, André, La Réglementation des métiers en Languedoc au Moyen Âge, Genève/Paris, Droz/Minard, 1958.

HEUSINGER (VON), Sabine, Die Zunft im Mittelalter : zur Verflechtung von Politik, Wirtschaft und Gesellschaft in Strassburg, Stuttgart, Steiner, 2009.

MCKENNEY, Richard, Tradesmen and traders: the world of the guilds in Venice and Europe, London, Barnes and Noble, 1987.

Les Métiers au Moyen Âge. Aspects économiques et sociaux. Actes du colloque international de Louvain-la-Neuve, 7-9 octobre 1993, dir. Pascale LAMBRECHTS et Jean-Pierre sosson, Louvainla-Neuve, Université catholique de Louvain, 1994.

Le Mouvement confraternel au Moyen Âge. France, Italie, Suisse, dir. Université de Lausanne, Roma, École française de Rome, 1987.

PINI, Antonio Ivan, L'Associazionismo medievale : comuni e corporazioni, Bologna, Consorzio provinciale pubblica lettura, 1974.

PINI, Antonio Ivan, Città, comuni e corporazioni nel Medioevo italiano, Bologna, Clueb, 1986.

RUTENBURG, Victor, « Arti e corporazioni », Storia d'Italia, Torino, Einaudi, 1973, vol. 5, t. I, p. 613-642.

1 Le Travail au Moyen Âge. Une approche interdisciplinaire, dir. Jacqueline HAMESSE et Colette MURAILLE-SAMARAN, Louvain-la-Neuve, Institut d'études médiévales, 1990.

2 Travail et travailleurs en Europe au Moyen Âge et aux débuts des temps modernes, dir. Claire DoLAN, Toronto, Pontifical Institute of Medieval Studies, 1991.

3 VINCENT, Catherine, Les Confréries médiévales dans le royaume de France: $\mathrm{XIII}^{e}-\mathrm{XV}^{e}$ siècle, Paris, Albin Michel, 1994.

WOLFF, Philippe et MAURO, Frédéric, L'Âge de l'artisanat ( $V^{e}-X V I I I^{e}$ s.), dans Histoire générale du travail (2), dir. Louis-Henri PARIAS, Paris, Nouvelle Librairie de France, 1960. 


\section{LES COMMUNAUTÉS TEXTUELLES}

CAMPBeL, Emma, Medieval Saint's Lives. The Gift, Kinship and community in old French hagiography, Cambridge, D. S. Brewer, 2008.

History in the comic mode, medieval communities and the matter of person, dir. Rachel FULTON et Bruce W. HOLSINGER, New York, Columbia University Press, 2007.

NEWHAUSER, Richard, The Seven Deadly Sins: from communities to individuals, Leiden/ Boston, Brill, 2007.

Rosenwein, Barbara H., Emotional communities in the early Middle Ages, Ithaca, Cornell University Press, 2006.

sтоск, Brian, The Implications of literacy: Written language and models of interpretation in the eleventh and twelfth centuries, Princeton, Princeton University Press, 1983.

\section{AUTEURS}

JULIA CONESA SORIANO

Université Paris-Sorbonne / Université Lyon II / Casa de Velázquez

JULIE PILORGET

Université Paris-Sorbonne 